\title{
A correction to "The retinal reference of the tilt aftereffect" by Coltheart and Cooper
}

\author{
N. J. WADE \\ The University, Dundee, Scotland \\ and \\ R. H. DAY \\ Monash University, Clayton 3168. Australia
}

\begin{abstract}
Coltheart and Cooper (1972) reexamined the data of Day and Wade (1969) concerning the retinal or gravitational reference for normalization and the tilt aftereffect. Their analysis contained a factual error concerning the procedure employed in the latter investigation, which is here corrected. Day and Wade did not, however, distinguish between normalization and aftereffect. When this distinction is made, the data from the two studies taken together indicate that deviation of an inspection line from the vertical retinal meridian is not sufficient to produce normalization but does vield a visual tilt aftereffect.
\end{abstract}

In reinterpreting our earlier data (Day \& Wade, 1969), Coltheart and Cooper (1972) make an error of fact, which is incorrectly attributed to one of us. They state: "The critical conditions in Day and Wade's experiment are Conditions III and IV. In Condition III an upright S made four pretest settings of a bar to the vertical. He was then tilted 15 deg clockwise and for 2 min inspected a gravitationally vertical bar. Then, with his body still tilted (N. J. Wade, personal communication), he made a single posttest setting to the gravitational vertical [p. 321: italics theirs]." A similar statement is made concerning Condition IV on p. 322. In actual fact, both Conditions III and IV of our study involved body tilt throughout the pretest, inspection, and posttest periods. This error renders their detailed reinterpretation of our data of little consequence.

Accepting the point about our failure to distinguish between normalization and the tilt aftereffect, it is of interest to reexamine our data in the light of Coltheart and Cooper's results. Adopting their nomenclature regarding the gravitational and retinal predictions for normalization and aftereffect [GN (gravitational normalization), RN (retinal normalization), GA (gravitational aftereffect), and RA (retinal aftereffect)], Condition III was addressed to the basis for normalization. The body was tilted $15 \mathrm{deg}$ right throughout the four pretest adjustments to the visual vertical, the 2 -min inspection of a gravitationally vertical line, and the single posttest setting to the visual vertical. Since the visual vertical was located relatively close to the gravitational vertical, Hypothesis GN wouid predict no effect, whereas RN would predict a reduction in the inclination of the visual vertical from the retinal meridian due to the normalization effect of the retinally tilted inspection figure. Small clockwise shifts of 0.73 and $0.64 \mathrm{deg}$ were measured in two experiments (Day \& Wade. 1969); these were attributed to postural adaptation, which has been demonstrated to have such an effect on visual verticality judgments during prolonged tilt (Wade, 1970). Thus, the absence of any normalization effect provides some support for the GN hypothesis, which is consonant with Prentice and Beardslee's (1950) analysis.

Condition IV was pertinent to the GA and RA hypotheses. The procedure was the same as for Condition III except that the inspection line was $15 \mathrm{deg}$ clockwise, in alignment with the body axis. A clockwise aftereffect would be predicted by GA; such would also be the case for RA, as a retinally vertical inspection figure generates a tilt aftereffect with a nonvertical test figure (Templeton, Howard, \& Easting, 1965). This point was overlooked in our interpretation and, therefore, this condition did not permit discrimination between the alternatives. Coltheart and Cooper, in demonstrating a tilt aftereffect with supine Ss, have provided evidence for the RA hypothesis:

Despite the factual error in Coltheart and Cooper's paper, a similar conclusion has been reached, due to the distinction between normalization and the tilt aftereffect. It appears that inclination of the inspection figure to the retinal vertical is a sufficient condition for the occurrence of a tilt aftereffect. whereas such is not the case for visual normalization. It remains to be firmly established whether inspection of a figure tilted with respect to gravity but not with respect to the retinal vertical is sufficient to produce normalization.

\section{REFERENCES}

Coltheart. M., \& Cooper. C. M. The retinal reference of the tilt aftereffect. Perception \& Psychophysics. 1972. 11,321-324. Day. R. H.. \& Wade. N. J. The reference for visual normalization. American Journal of Psychology 1969. 82, 191-197.

Prentice. IV C. H.. \& Bearslee. D. C. Visual "normalization" near 
the vertical and horizontal. Journal of Experimental Psychology, 1950, 40, 355-364.

Templeton, W. B., Howard, I. P.. \& Easting, G. Satiation and the tilt aftereffect. American Journal of Psychology, 1965, 78, 656-659.

Wade, N. J. The effect of prolonged tilt on visual orientation.
Quarterly Journal of Experimental Psychology. 1970. 22. 423-439.

(Received for publication November 6, 1972: revision received December 7,1972 .) 